\title{
High resolution melting (HRM) analysis for genetic changes in BRCA1/2 gene
}

\author{
Samuel J Haryono ${ }^{1,2 *}$, I Gusti Bagus Datasena², Ariananda Hariadi², Raymond \\ Mulyarahardja ${ }^{2}$ \\ ${ }^{1}$ Departments of Oncology Surgery \\ 2 Vivo Research Initiative \\ DOI: http://dx.doi.org/10.19106/JMedScieSup004804201623
}

\section{ABSTRACT}

Conventional mutation analysis requires a separation step and includes singlestrand conformational polymorphism (SSCP) analysis, denaturing (denaturation of) gradient gel electrophoresis, heteroduplex analysis, denaturing(denaturation of) HPLC, and temperature gradient capillary electrophoresis These methods require separation of PCR products on gel or other matrix, which often take hours to perform and increase the risk of contamination in future reactions as they are exposed to the environment. High Resolution Melting (HRM) might simplify the mutation scanning analysis in BRCA $1 / 2$ gene. DNA from affected patients and family members were amplified with Real-Time PCR reaction and followed by Sanger Sequencing to reconfirm the mutation status obtained by HRM Method. HRM Method was able to show distinction in differential curves of mutated BRCA 2 gene c.4600T>C, with codon modification of CAT>TAT, when compared to wildtype. This method requires two groups of experimental standards and standard curves to determine point mutation in the sample. The first standard was taken from samples without mutation (wildtype/negative control) and the second standard from samples with mutation (positive control), both were confirmed by Sanger Sequencing. The sequencing analysis of the affected patient and the family members showed that mutation occurred (BRCA2 c.4600T $>$ C), causing amino acid alteration in BRCA2 protein (p.H1458Y), and was segregated in the family history. HRM Method is an excellent tool to analyze genetic modification of BRCA $1 / 2$ genes, especially in investigating the co-segregation of mutated genes among family members of affected individual. This method might also provide more sensitive results to determine mutation in patient before using Sanger Sequencing analysis.

Keyword: BRCA, HRM, Gene Mutation 